\title{
Evaluation of Biochemical Recurrence-free Survival after Radical Prostatectomy by Cancer of the Prostate Risk Assessment Post-Surgical (CAPRA-S) Score
}

\author{
Binhan Kagan Aktas*, Cuneyt Ozden, Suleyman Bulut, Suleyman Tagci, Guven \\ Erbay, Cevdet Serkan Gokkaya, Mehmet Murat Baykam, Ali Memis
}

\begin{abstract}
Background: The cancer of the prostate risk assessment (CAPRA) score has been defined to predict prostate cancer recurrence based on the pre-clinical data, then pathological data have also been incorporated. Thus, CAPRA post-surgical (CAPRA-S) score has been developed based on six criteria (prostate specific antigen (PSA) at diagnosis, pathological Gleason score, and information on surgical margin, seminal vesicle invasion, extracapsular extension and lymph node involvement) for the prediction of post-surgical recurrences. In the present study, biochemical recurrence $(B C R)$-free probabilities after open retropubic radical prostatectomy (RP) were evaluated by the CAPRA-S scoring system and its three-risk level model. Materials and Methods: CAPRA-S scores (0-12) of our 240 radical prostatectomies performed between January 2000-May 2011 were calculated. Patients were distributed into CAPRA-S score groups and also into three-risk groups as low, intermediate and high. BCR-free probabilities were assessed and compared using Kaplan-Meier analysis and Cox proportional hazards regression. Ability of CAPRA-S in BCR detection was evaluated by concordance index (c-index). Results: BCR was present in 41 of total 240 patients $(17.1 \%)$ and the mean follow-up time was $51.7 \pm 33.0$ months. Mean BCR-free survival time was 98.3 months (95\% CI: 92.3-104.2). Of the patients in low, intermediate and high risk groups, $5.4 \%, \mathbf{2 2 . 0 \%}$ and $58.8 \%$ had $\mathrm{BCR}$, respectively and the difference among the three groups was significant $(P=0.0001) . C$-indices of CAPRA-S score and three-risk groups for detecting BCR-free probabilities in 5-yr were 0.87 and 0.81 , respectively. Conclusions: Both CAPRA-S score and its three-risk level model well predicted BCR after RP with high c-index levels in our center. Therefore, it is a clinically reliable post-operative risk stratifier and disease recurrence predictor for prostate cancer.
\end{abstract}

Keywords: Nomogram - prostate cancer - radical prostatectomy - recurrence - survival

Asian Pac J Cancer Prev, 16 (6), 2527-2530

\section{Introduction}

Prostate cancer is the second most commonly seen cancer in Turkish men, other than non-melanoma skin cancer and has an age adjusted incidence rate of 22.8 per 100,000 men (Eser et al., 2010). Radical prostatectomy (RP) is the most promising current treatment option for localized prostate cancer (Hu et al., 2014). About one third of patients in whom prostate specific antigen (PSA) value was initially dropped down to zero after the operation would have biochemical recurrence (BCR), which is defined as two or more consecutive increase of serum PSA values $>0.2 \mathrm{ng} / \mathrm{mL}$ (Moul, 2000).

The prediction of the likelihood of BCR is critical for surveillance strategy and risk stratification after RP. Like several previously published prognostic instruments, the University of California, San Francisco, Cancer of the Prostate Risk Assessment (UCSF-CAPRA), also widely known as CAPRA score, was proposed in 2005, to help predict the individualized risk of BCR after RP. The CAPRA score relies on five preoperative variables including PSA, biopsy Gleason score, clinical stage, percentage of positive biopsy, and age at diagnosis (Cooperberg et al., 2005). Six years afterwards in 2011 the same institute developed the CAPRA post-surgical (CAPRA-S) score to improve the accuracy of prediction of BCR by incorporating pathologic information (Cooperberg et al., 2011). Studies including large, multiinstitutional and comparative trials have externally validated CAPRA-S score and confirmed BCR prediction after RP (Lughezzani et al., 2010; Punnen et al., 2014).

Given the worldwide disparities in patient characteristics, we aimed to examine the validity of the CAPRA-S score in our cohort consisting of prostate cancer patients at a single institution in Turkey and to provide a survival data on prostate cancer in Turkish patients. 


\section{Materials and Methods}

Between January 2000 and May 2011, in urology clinic of a tertiary care teaching hospital, clinically localized prostate cancer patients for whom open retropubic RP was performed as the sole treatment were included in the present retrospective study. Patients who received any neoadjuvant treatment, and who underwent adjuvant therapy before BCR were not included. The study was approved by applicable local ethics committee. Informed consent was not obtained due to the retrospective nature of the study. A total of 240 patients had proper data to calculate the CAPRA-S score which has a final sum between $0-12$ by addition of the points of each six variables including PSA at diagnosis, pathology Gleason score, surgical margin (SM) status, and the presences of seminal vesicle invasion (SVI), extracapsular extension (ECE), and lymph node involvement (LNI). Scores $\geq 9$ were combined as originally described (Cooperberg et al., 2011).

Then the patients were distributed into three-risk level groups according to CAPRA-S scores: 0-2 as low, 3-5 as intermediate, and $\geq 6$ as high risk. Two consecutive increases of serum PSA $>0.2 \mathrm{ng} / \mathrm{mL}$, two weeks apart were defined as BCR following RP.

\section{Statistical analysis}

IBM statistical package for social sciences (IBM Co., Armonk, NY, USA) version 21.0 software was used for the analysis of data. The data expressed as the mean \pm standard deviation. The performances of the each CAPRA-S score groups and the three-risk level model to predict the 3- and 5-yr BCR-free probability following RP were examined by Cox proportional hazards regression and KaplanMeier analysis. Harrel's concordance index (c-index) was calculated to evaluate the 3- and 5-yr prediction probabilities of CAPRA-S score and the three-risk level model, indicating a perfect concordance with values closer to 1.0 (Harrell et al., 1996).

\section{Results}

Mean levels of the patients were $62.7 \pm 6.2$ years for age, $10.9 \pm 6.9 \mathrm{ng} / \mathrm{mL}$ for PSA and $46.7 \pm 23.5 \mathrm{~mL}$ for prostate volume. Distribution of the data regarding level
Table 1. Distribution of the Data Regarding Level of the 6 Variables Constituting CAPRA-S Scoring System

\begin{tabular}{llcr}
\hline Variable & Level & Points & No. $(\%)$ \\
\hline PSA $(\mathrm{ng} / \mathrm{mL})$ & $0-6$ & 0 & $59(24.6 \%)$ \\
& $6.01-10$ & 1 & $80(33.3 \%)$ \\
& $10.01-20$ & 2 & $75(31.3 \%)$ \\
& $>20$ & 3 & $26(10.8 \%)$ \\
SM & Negative & 0 & $199(82.9 \%)$ \\
& Positive & 2 & $41(17.1 \%)$ \\
SVI & No & 0 & $212(88.3 \%)$ \\
& Yes & 2 & $28(11.7 \%)$ \\
Gleason Score & $2-6$ & 0 & $177(73.8 \%)$ \\
& $3+4$ & 1 & $18(7.5 \%)$ \\
& $4+3$ & 2 & $22(9.2 \%)$ \\
& $8-10$ & 3 & $23(9.6 \%)$ \\
ECE & No & 0 & $192(80.0 \%)$ \\
LNI & Yes & 1 & $48(20.0 \%)$ \\
& No & 0 & $231(96.3 \%)$ \\
& Yes & 1 & $9(3.7 \%)$ \\
\hline
\end{tabular}

Table 2. BCR-free Probabilities for Each CAPRA-S Score Group and the Three-Risk Level Model in 5-yr

\begin{tabular}{cccc}
\hline \multicolumn{2}{l}{ Score/Risk level p value } & HR $(95 \% \mathrm{CI})$ & $\begin{array}{c}\text { 5-yr BCR-free } \\
\text { probability }(95 \% \mathrm{CI})\end{array}$ \\
\hline \multicolumn{4}{c}{ CAPRA-S score groups } \\
$0-2$ & ref & 1 & $92.9(88.0-97.8)$ \\
3 & 0.86 & $1.2(0.3-5.5)$ & $91.8(81.0-100.0)$ \\
4 & $<0.001$ & $8.4(3.1-22.3)$ & $60.2(37.3-83.1)$ \\
5 & $<0.001$ & $8.9(2.4-33.7)$ & $59.3(23.0-95.6)$ \\
6 & $<0.001$ & $14.1(5.1-38.9)$ & $42.2(14.2-70.2)$ \\
7 & $<0.001$ & $10.4(2.2-49.3)$ & $50.0(1.0-99.0)$ \\
8 & $<0.001$ & $30.3(7.9-116.2)$ & $25.0(0.0-67.5)$ \\
$>9$ & $<0.001$ & $23.7(8.8-63.9)$ & 0 \\
CAPRA-S risk levels & & \\
Low & ref & 1 & $92.9(88.0-97.8)$ \\
Intermediate & $<0.001$ & $4.3(1.8-10.5)$ & $75.9(63.9-87.9)$ \\
High & $<0.001$ & $17.5(7.7-40.0)$ & $28.8(11.7-45.9)$ \\
\hline
\end{tabular}

of the six variables constituting CAPRA-S scoring system is shown in Table 1. There were 147 (61.2\%), 59 (24.6\%) and $34(14.2 \%)$ patients in low, intermediate and high CAPRA-S risk levels, respectively. The distribution of patients into each CAPRA-S score groups (from 0 to >9) was, respectively, as follows (patients, \%): 41 (17.1\%); 52 (21.7\%); 54 (22.5\%); 28 (11.7\%); 21 (8.7\%); 10 (4.2 $\%) ; 14(5.8 \%) ; 6(2.5 \%) ; 4(1.7 \%)$ and $10(4.1 \%)$. BCR was detected in a total of 41 patients $(17.1 \%)$ at a mean

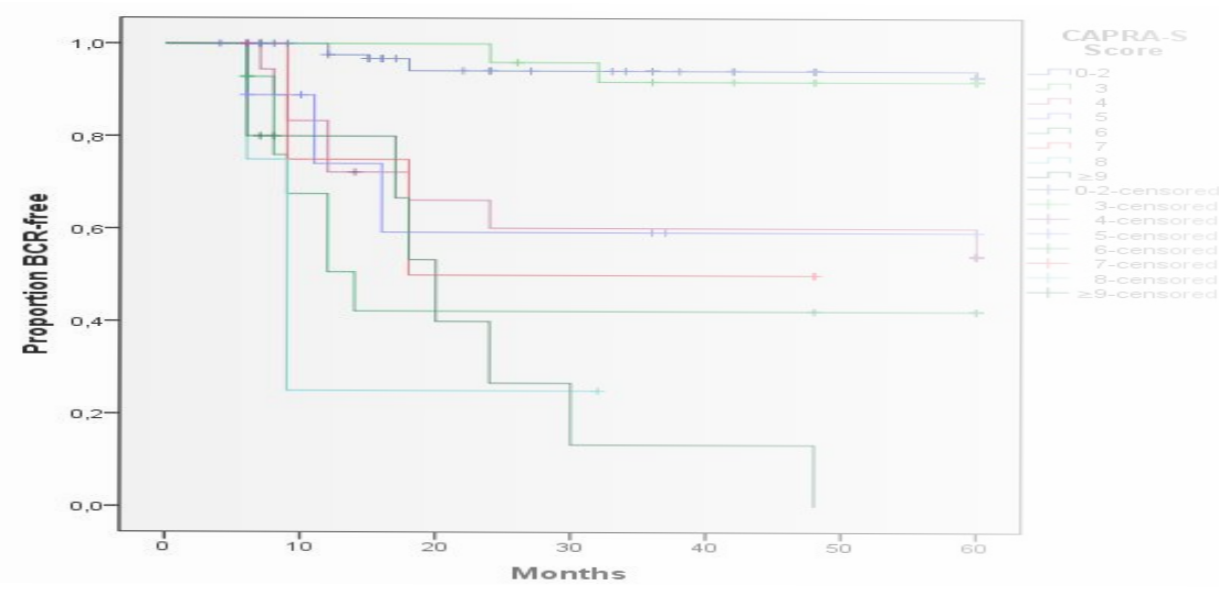

Figure 1. Kaplan-Meier Curves of CAPRA-S Score Groups Showing 5-yr BCR-free Survival Rates after RP 
Table 3. Concordance Indices of CAPRA-S Score Groups and Three-Risk Level Model for 3- and 5-yr

\begin{tabular}{llc}
\hline Time & \multicolumn{1}{c}{ Variables } & C-index (95\% CI) \\
\hline 3 -yr & CAPRA-S score groups & $0.86(0.79-0.92)$ \\
& Three-risk level model & $0.80(0.71-0.88)$ \\
5 -yr & CAPRA-S score groups & $0.87(0.81-0.93)$ \\
& Three-risk level model & $0.81(0.72-0.89)$ \\
\hline
\end{tabular}

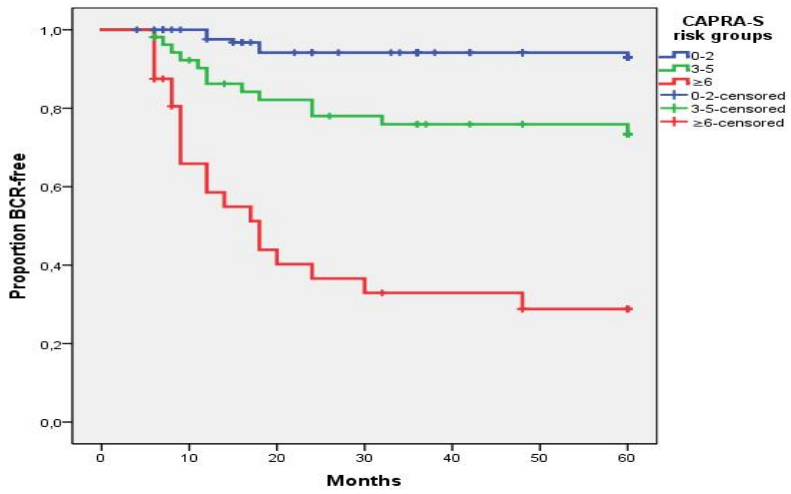

Figure 2. Kaplan-Meier Curves of CAPRA-S threeRisk Groups Showing 5-yr BCR-free Survival Rates after RP

$51.71 \pm 32.98$ month follow-up. The mean BCR-free survival time was 98.3 months (95\%CI: 92.3-104.2). Eight of 147 (5.4\%), 13 of $59(22.0 \%)$ and 20 of 34 (58.8\%) patients in low, intermediate and high risk groups, respectively had BCR and the difference was significant $(\mathrm{p}=0.0001)$. The 5 -yr BCR-free survival rate for all 240 patients was 79.5\% (95\% CI: 73.8- 85.2). BCR-free probabilities and hazard ratios for each CAPRA-S score group and the three-risk level model in 5-yr are tabulated in Table 2 and the Kaplan-Meier curves are presented in Figure 1 and 2. C-indices of CAPRA-S score and threerisk level groups for 3- and 5-yr are shown in Table 3.

\section{Discussion}

It can be hard to decide for clinicians which one is ideal to use among the $>100$ published predictive tools addressing different oncological outcomes for prostate cancer patients (Shariat et al., 2008). Considering the difference in baseline characteristics of patients influenced mainly by race and ethnicity, the situation becomes more and more complex. Recently, a scoring system named CAPRA-S which base on pathological data on RP specimen gained popularity owing to its relatively high concordance value of 0.77 (Cooperberg et al., 2011). In external validations, a Korean study reached the same c-index level as originally reported (Seong et al., 2013). In the study of Punnen et al. including a collection of data from multiple institutions across United States, which is called as Shared Equal Access Regional Cancer Hospital (SEARCH) database, CAPRA-S was found to be slightly superior to the commonly known and referenced nomogram developed by Stephenson et al. (the c-index levels were 0.73 and 0.72 , respectively) (Punnen et al., 2014). And in a more recent study also from Korea, Seo et al. reported c-indices as high as 0.80 (Seo et al., 2014). Suprisingly in our study, we have detected c-indices over 0.80 for both 3- and 5-yr BCR-free probabilities. Besides, c-indices remained over 0.80 without depending on which score group (each CAPRA-S score groups or three-risk level model) we used. These results indicated that CAPRA-S score and its three-risk level model well predicted the post-operative BCR in our cohort consisting of Turkish prostate cancer patients.

The comparison of our 5-yr BCR-free probabilities for each CAPRA-S score with those of SEARCH, Cancer of the Prostate Strategic Urologic Research Endeavor (CaPSURE) (Cooperberg et al., 2011) and Korean data set (Seo et al., 2014) showed that our values are higher than SEARCH and CaPSURE, but lower than Korean data. Since our BCR rate of $17.1 \%$ is higher than that of Korean (13.8\%) and lower than that of SEARCH (34.3\%), this result might be derived from different BCR rates. However, in comparison of our BCR-free probabilities with those of CaPSURE, which has a BCR rate of $16.8 \%$, there is a discrepancy in spite of the proximity between $\mathrm{BCR}$ rates. Our BCR-free probabilities were expected to be slightly lower than those of CaPSURE. Contrary to this expectation, we had apparently higher values. We think that a reasonable explanation of this discrepancy might lie within the racial and ethnic disparities. The results of the study undertaken by Tyson and Castle demonstrating remarkable racial disparities in a large American cohort, support our theory (Tyson and Castle, 2014).

Considering our data according to distribution of patients into CAPRA-S risk groups, we have detected the highest frequency in the low risk group as in CaPSURE and SEARCH data sets. However, in two Korean studies, the patients in intermediate risk groups $(44.6 \%$ for Seo et al. and $31.3 \%$ for Seong et al.) and in high risk groups ( $25.4 \%$ for Seo et al. and $35.8 \%$ for Seong et al.) comprised higher proportions of the total, compared with our study (24.6\% and $14.2 \%$ in intermediate and high risk groups, respectively). This result might be derived from higher exposure of our patients to PSA screening. Although a small, regional study claimed that Turkish patients were reluctant to prostate cancer screening (Ceber et al., 2008), in the multi-center study of Zorlu et al. on the epidemiology of prostate cancer, it was demonstrated that $54.4 \%$ of patients had clinical T1c cancer in Turkey (Zorlu et al., 2014). The frequency level of T1c patients was $52.5 \%$ in the present study and this result supports our hypothesis. And, the results of Kang et al. demonstrating worse disease characteristics of Korean prostate cancer patients compared to their American counterparts, point out the racial differences as another contributing factor for that difference (Kang et al., 2013).

It is expected that BCR-free probabilities would decrease with increasing CAPRA-S score. With the exception of CAPRA-S score 7 group which has an unexpectedly high BCR-free probability, expected results that are suitable to the risk level of each group, are obtained in the present study. In the study of Seo et al. conducted in 130 patients, progression-free probabilities for CAPRA-S score 5 and 6 groups were disproportional to other score groups (Seo et al., 2014). However, there was not any such problematic values in large CaPSURE (Cooperberg et al., 2011) and SEARCH data sets (Punnen et al., 2014). 


\section{Binhan Kagan Aktas et al}

Therefore, we think that this is a limitation arising from small number of patients. Unlike each CAPRA-S score groups, the three-risk level model was not affected by this limitation. BCR-free probabilities decreased and hazard ratios increased in concordance with increasing risk in the present study. It was also found that BCR-free survival of three-risk level model was significantly different from each other in survival analysis.

In conclusion, CAPRA-S score and its more practical form according to risk levels accurately predicted BCR after RP with high c-index levels in our patients. It is quite satisfactory to use the present form of CAPRA-S scoring system on our cohort and it seems that no specific revision is needed for Turkish prostate cancer patients. It is an easy to use and non time-consuming clinical tool, which can even be calculated from memory without a need for a computer or paperwork.

\section{References}

Ceber E, Cakir D, Ogce F, et al (2008). Why do men refuse prostate cancer screening? Demographic analysis in Turkey. Asian Pac J Cancer Prev, 9, 387-90.

Cooperberg MR, Pasta DJ, Elkin EP, et al (2005). The University of California, San Francisco Cancer of the Prostate Risk Assessment Score: a straightforward and reliable preoperative predictor of disease recurrence after radical prostatectomy. J Urol, 173, 1938-42.

Cooperberg MR, Hilton JF, Carroll PR (2011). The CAPRA-S score: a straightforward tool for improved prediction of outcomes after radical prostatectomy. Cancer, 117, 5039-46.

Eser S, Yakut C, Ozdemir R, et al (2010). Cancer incidence rates in Turkey in 2006: a detailed registry based estimation. Asian Pac J Cancer Prev, 11, 1731-9.

Harrell FE Jr, Lee KL, Mark DB (1996). Multivariable prognostic models: issues in developing models, evaluating assumptions and adequacy, and measuring and reducing errors. Stat Med, 15, 361-87.

Hu XH, Cammann H, Meyer HA, et al (2014). Risk prediction models for biochemical recurrence after radical prostatectomy using prostate-specific antigen and Gleason score. Asian $J$ Androl, published online.

Kang DI, Chung JI, Ha HK, et al (2013). Korean prostate cancer patients have worse disease characteristics than their American counterparts. Asian Pac J Cancer Prev, 14, 6913-7.

Lughezzani G, Budaus L, Isbarn H, et al (2010). Head-to-head comparison of the three most commonly used preoperative models for prediction of biochemical recurrence after radical prostatectomy. Eur Urol, 57, 562-8.

Moul JW (2000). Prostate specific antigen only progression of prostate cancer. J Urol, 163, 1632-42.

Punnen S, Freedland SJ, Presti JC Jr, et al (2014). Multiinstitutional validation of the CAPRA-S score to predict disease recurrence and mortality after radical prostatectomy. Eur Urol, 65, 1171-7.

Seo WI, Kang PM, Kang DI, et al (2014). Cancer of the prostate risk assessment (CAPRA) preoperative score versus postoperative score (CAPRA-S): ability to predict cancer progression and decision-making regarding adjuvant therapy after radical prostatectomy. J Korean Med Sci, 29, 1212-6.

Seong KT, Lim JH, Park CM, Kim HK, Park JY (2013). External validation of the cancer of the prostate risk assessment-s score in Koreans undergoing radical prostatectomy. Korean J Urol, 54, 433-6.
Shariat SF, Karakiewicz PI, Roehrborn CG, Kattan MW (2008). An updated catalog of prostate cancer predictive tools. Cancer, 113, 3075-99.

Tyson MD 2nd, Castle EP (2014). Racial disparities in survival for patients with clinically localized prostate cancer adjusted for treatment effects. Mayo Clin Proc, 89, 300-7.

Zorlu F,Zorlu R, Divrik RT, Eser S, Yorukoglu K (2014). Prostate cancer incidence in Turkey: an epidemiological study. Asian Pac J Cancer Prev, 15, 9125-30. 